Original article

\title{
PRECEDE-PROCEED model based questionnaire and saliva tests for oral health checkup in adult
} \author{
and Nobuhiro Hanada ${ }^{1)}$ \\ ${ }^{1)}$ Department of Translational Research, Tsurumi University School of Dental Medicine, Yokohama, Japan
${ }^{2)}$ Kawasaki Dental Association, Kawasaki, Japan \\ ${ }^{3)}$ Fukai Institute of Health Science, Misato, Japan
}

Yoshiaki Nomura ${ }^{1)}$, Tomoaki Matsuyama ${ }^{2}$, Kakuhiro Fukai $^{3)}$, Ayako Okada ${ }^{1)}$, Mitsuo Ida $^{2}$, Noriaki Yamauchi $^{2}$, Hiroyuki Hanamura ${ }^{2)}$, Yoshihide Yabuki ${ }^{2)}$, Kazuhiko Watanabe ${ }^{2}$, Motoko Sugawara ${ }^{2}$, Yasuhiko Imanishi' ${ }^{2}$, Norihiro Koizumi ${ }^{2)}$, Yoshinori Murano ${ }^{2}$, Atsushi Nishiyama ${ }^{2)}$, Yoshiteru Fukukawa ${ }^{2}$, Ryoko Otsuka ${ }^{1}$,

(Received August 21, 2018; Accepted January 9, 2019)

\begin{abstract}
Screening is a fundamental strategy for early detection, treatment, and prevention of progression of oral disease and those at high risk for oral disease. While numerous screening tools exist, questionnaires, and saliva tests are often suitable for screening. The questionnaire based on the PRECEDE-PROCEED model was developed, validated, and elucidated on the structural interrelationship between these two methods. In the current investigation, 311 adults had this questionnaire and saliva testing administered simultaneously during an occupational health checkup. The questionnaire was validated by classical test theory, item response theory, and path analysis. Through structural equation modeling, it was found that self-care guidance may be an important role of the family dentist. In addition, self-awareness of oral symptoms was significantly related to saliva test results. However, self-administered questionnaires and saliva tests together may provide more information than either of them alone for the detection, treatment, and prevention of progression of oral disease. Thus, simultaneous application of self-administered questionnaires and saliva tests is recommended during oral health checkups for adults.
\end{abstract}

Keywords; healthy people programs, oral health, mass screening, saliva, health surveys

\section{Introduction}

Dental and oral health are essential for maintaining good food intake [1]. Lifelong maintenance of healthy oral function greatly contributes to the prevention of disease. In addition, recent work has shown that periodontal disease can increase the risk for a wide variety of noncommunicable diseases (NCDs). Thus, early detection and treatment of periodontal disease may lead to the prevention of NCDs [2].

Aimed at providing a sustainable social security system for the aging society of Japan, the Law Concerning the Security of Healthcare Treatment for Senior Citizens (Law No. 80, 1982) was enacted. Provisions of this law, specific health checkups (SHC) and specific health guidance (SHG), have been implemented targeting middle-aged adults who will become elderly at a time when the proportion of elderly people is projected to be the largest. The purpose of SHC and SHG is the prevention of metabolic syndrome as a representative NCD. This checkup system is widespread in Japan, and insurers of national coverage health insurance (i.e., companies and local governments) are obliged to carry out this checkup system. However, to date, this checkup system has not included oral health checkups.

Checkups for oral health should be included in a systematic health checkup system. The primary obstacles to including oral checkups are the cost and time associated with oral examinations performed by a dentist. With this in mind, the Japan Dental Association developed and distributed an oral health questionnaire based on the PRECEDE-PROCEED model.

Correspondence to Dr. Yoshiaki Nomura, Department of Translational Research, Tsurumi University School of Dental Medicine, 2-1-3 Tsurumi, Tsurumi-ku, Yokohama, Kanagawa 230-8501, Japan Fax: +81-45-573-2473 E-mail: nomura-y@tsurumi-u.ac.jp

J-STAGE Advance Publication: September 21, 2019

Color figures can be viewed in the online issue at J-STAGE.

doi.org/10.2334/josnusd.18-0288

DN/JST.JSTAGE/josnusd/18-0288
This questionnaire can be systematically included in and may be useful for SHC and SHG [3]. In addition, saliva tests are noninvasive, can be carried out without any dental professionals, and have been developed for periodontal disease screening [4]. Salivary lactate dehydrogenase (LD) level, a result from this test, has been reported to correlate with individual's medical expenses [5]. Therefore, saliva tests may be a useful candidate for systematic health checkup systems.

While many oral health checkup tools exist, the aforementioned methods are good SHC and SHG item candidates. Thus, it was necessary to elucidate the relationship between these two oral health checkup methods. In this study, the PRECEDE-PROCEED model questionnaire and saliva tests were simultaneously administered to adults at an occupational health checkup. The validity of the questionnaire based on the PRECEDE-PROCEED model was confirmed and the structural interrelationship of these two methods was analyzed.

\section{Materials and Methods}

\section{Subjects}

Three hundred eleven adult office workers that had undertaken occupational health checkups from March 2016 to February 2017 were included in this study. Subjects who used removable dentures were excluded. The study population consisted of 187 men and 124 women. Their mean age was $47.0 \pm 14.0$ years.

\section{Questionnaire}

The questionnaire used in this study was developed by the Japan Dental Association as part of The Adult Oral Health Assessment and Instruction Program. The questionnaire consisted of 20 items [3], which were selected based on the framework of the PRECEDE-PROCEED model [6].

\section{Saliva test}

According to the manufacturer's (Eiken Kagaku) instructions, saliva samples were collected at least $2 \mathrm{~h}$ after eating, drinking, or teeth brushing. Participants were instructed to chew on a standardized tasteless and odorless gum base for $5 \mathrm{~min}$, and the stimulated whole saliva was continuously collected. To examine salivary levels of Hemoglobin (Hb), $100 \mu \mathrm{L}$ of the saliva was immediately transferred into a diluent solution containing preservative and kept at $4^{\circ} \mathrm{C}$. Additional saliva for measuring $\mathrm{LD}$ was also kept at $4^{\circ} \mathrm{C}$. Salivary levels of $\mathrm{Hb}$ and LD were measured using commercially available kits (OC-HEMODIA AUTO S; Eiken Kagaku, Tokyo, Japan, and L type Wako LDH J; Wako Chemical Industry, Osaka, Japan) according to the manufacturer's instructions [7-9].

\section{Statistical analysis}

Distributions of the response to the questionnaire were summarized in a frequency table, and $\varphi$ coefficients (also referred to discrimination index) of each item were calculated. To compare the saliva test results against the items of the questionnaire, the Mann-Whitney $U$ test was applied. For item response curves and item formation function, two-parameter logistic models were used. Principal component analysis was carried out for the factors that corresponded to the constituents of PRECEDE-PROCEED model. First main principal component scores of the following contents: 
Table 1 Descriptive statistics for the PRECEDE-PROCEED model based questionnaire used in this study

\begin{tabular}{|c|c|c|c|c|c|c|}
\hline \multirow[t]{2}{*}{ No } & \multirow[t]{2}{*}{ Item } & \multirow[t]{2}{*}{ Answer } & \multicolumn{2}{|c|}{$\begin{array}{l}\text { Number of subjects } \\
\text { answering Yes }\end{array}$} & \multirow{2}{*}{$\begin{array}{c}\varphi \\
\text { coefficient }\end{array}$} & \multirow[t]{2}{*}{$P$ value } \\
\hline & & & $n$ & $\%$ & & \\
\hline Q1 & Do you have any concerns about your teeth or mouth? & Yes/No & 188 & 60.5 & 0.487 & $<0.001$ \\
\hline Q2 & $\begin{array}{l}\text { If you answered "Yes" to Question 1, what type of concern do you have? } \\
\text { 1) Difficulty chewing 2) Poor appearance of teeth/mouth } \\
\text { 3) Speech problems 4) Bad Breath } \\
\text { 5) Tooth/oral pain 6) Other ( ) }\end{array}$ & & 28 & 9.0 & 0.204 & 0.636 \\
\hline Q3 & Can you bite with your back teeth? & Yes/No & 55 & 17.7 & 0.344 & $<0.001$ \\
\hline Q4 & Do your gums bleed when you brush your teeth? & $\mathrm{No} / \mathrm{Yes}$ & 136 & 43.7 & 0.431 & $<0.001$ \\
\hline Q5 & Do you have swollen gums? & $\mathrm{No} / \mathrm{Yes}$ & 63 & 20.3 & 0.360 & $<0.001$ \\
\hline Q6 & Do your teeth hurt when you consume cold or hot things? & $\mathrm{No} / \mathrm{Yes}$ & 137 & 44.1 & 0.433 & $<0.001$ \\
\hline Q7 & Do you have a dentist that you consider to be your personal or family dentist? & No/Yes & 86 & 27.7 & 0.504 & $<0.001$ \\
\hline Q8 & Are there times when you are too busy to go to the dentist? & No/Yes & 158 & 50.8 & 0.489 & $<0.001$ \\
\hline Q9 & $\begin{array}{l}\text { Are you currently undergoing treatment for any of the following diseases or } \\
\text { medical conditions? }\end{array}$ & Yes/No & 20 & 6.40 & 0.225 & 0.448 \\
\hline Q10 & Are the people close to you concerned about daily oral health or oral care? & $\mathrm{No} / \mathrm{Yes}$ & 123 & 39.5 & 0.432 & $<0.001$ \\
\hline Q11 & $\begin{array}{l}\text { Do you feel confident about your teeth, or do people sometimes complain about } \\
\text { your teeth? }\end{array}$ & No/Yes & 271 & 87.1 & 0.311 & $<0.001$ \\
\hline Q12 & Do you usually brush your teeth at work or when you are out? & Yes/No & 243 & 78.1 & 0.416 & $<0.001$ \\
\hline Q13 & Do you consume sweet drinks between meals? & Yes/No & 266 & 85.5 & 0.281 & 0.046 \\
\hline Q14 & Do you smoke? & Yes/No & 65 & 20.9 & 0.357 & $<0.001$ \\
\hline Q15 & Do you brush your teeth before bed? & $\mathrm{No} / \mathrm{Yes}$ & 73 & 23.5 & 0.415 & $<0.001$ \\
\hline Q16 & Do you use fluoride toothpaste? & Yes/No & 167 & 53.7 & 0.318 & 0.004 \\
\hline Q17 & Do you use floss or an interdental brush? & Yes/No & 244 & 78.5 & 0.427 & $<0.001$ \\
\hline Q18 & Do you chew your food slowly and carefully when you eat? & Yes/No & 246 & 79.1 & 0.384 & $<0.001$ \\
\hline Q19 & Have you ever received tooth brushing instruction at a dental clinic or elsewhere? & Yes/No & 104 & 33.4 & 0.430 & $<0.001$ \\
\hline Q20 & Do you visit a dental clinic for a regular checkup at least once a year? & No/Yes & 172 & 55.3 & 0.493 & $<0.001$ \\
\hline
\end{tabular}

The PRECEDE-PROCEED model based questionnaire used in this study. It is developed by Japan Dental Association and is distributed freely. This questionnaire was developed for specified health checkups and specified health guidance.

For Q2, number of subjects and percentage of subject indicate number of subjects at least answered one sub item.

(A)

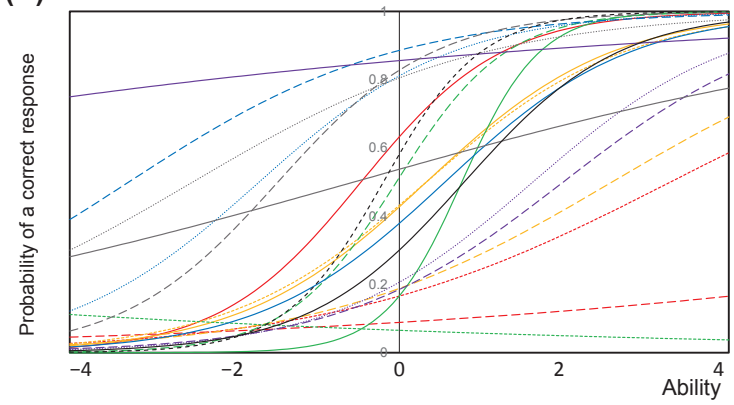

Q1 Do you have any concerns about your teeth or mouth?

------ Q3 Can you bite with your back teeth?

Q5 Do you have swollen gums?

Q7 Do you have a dentist that you consider to be your personal or family dentist?

-.-.--- Q9 Are you currently undergoing treatment for any of the following diseases or medical conditions?

- - Q11 Do you feel confident about your teeth, or do people sometimes complain about your teeth?

Q13 Do you consume sweet drinks between meals?

............ Q15 Do you brush your teeth before bed?

$---Q 17$ Do you use floss or an interdental brush?

Q19 Have you ever received tooth brushing instruction at a dental clinic or elsewhere?
(B)

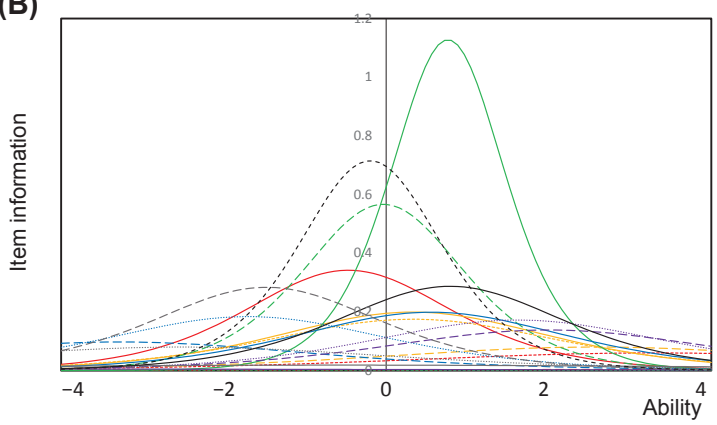

- - Q2 If you answered "Yes" to Question 1, what type of concern do you have ?

Q Q4 Do your gums bleed when you brush your teeth?

Q6 Do your teeth hurt when you consume cold or hot things?

- - - Q8 Are there times when you are too busy to go to the dentist?

Q10 Are the people close to you concerned about daily oral health or oral care?

……..... Q12 Do you usually brush your teeth at work or when you are out?

- - - Q14 Do you smoke?

Q16 Do you use fluoride toothpaste?

............. Q18 Do you chew your food slowly and carefully when you eat?

- - - Q Q20 Do you visit a dental clinic for a regular checkup at least once a year?

Fig. 1 Item response curves and item information functions of the PRECEDE-PROCEED model based questionnaire. Horizontal axis indicates the unhealthiness. (A)Item response curve: Most of the items of PRECEDE-PROCEED model based questionnaire were useful to evaluate the unhealthiness. (B)Item information function: Item of Q7: having family dentist or not had the highest information. This item was useful for evaluating health.

"Health," "Behavior," "Environment," "Reinforcing Factors," and "Enabling Factors" were used for path analysis. In addition, factor analysis with varimax rotation was carried out. Structural equation modeling was used to create path diagrams for items extracted by factor analysis and the results of saliva test. $P$ values less than 0.05 were considered statistically significant. These procedures were carried out using SPSS Statistics Ver 24.0 and AMOS Ver 24.0 (IBM, Tokyo, Japan). For analysis of item response theory, R software (ltm and irtoys packages) was used.

\section{Ethics}

All the procedures were conducted in accordance with the Declaration of Helsinki. Before saliva sampling, informed consent was obtained from each subject that participated in this study. This study was approved by the ethical committee of the Tsurumi University School of Dental Medicine (Approval number: 1601).

\section{Results}

The distributions and $\varphi$ coefficients of the items of the questionnaire are shown in Table 1. Regarding the reliability of the items in the questionnaire, Cronbach's coefficient alpha was 0.664 . The items with high $\varphi$ coefficients were family dentist (Q7, enabling factors), regular checkups (Q20, behavior), spare time to visit dentist (Q8, enabling factors), and subjective symptoms (Q1, QOL).

Figure 1 shows the item response curves calculated by the two-parameter logistic model. In the item response curve, the steepness of the curve at its inflection point provides a measure of the discriminatory power of the item. The discrimination of the item refers to how well an item can distinguish between respondents with low ability levels versus those with high ability levels. A steep item response curve indicates that the item has strong discrimination. Respondents with low ability levels have a low 


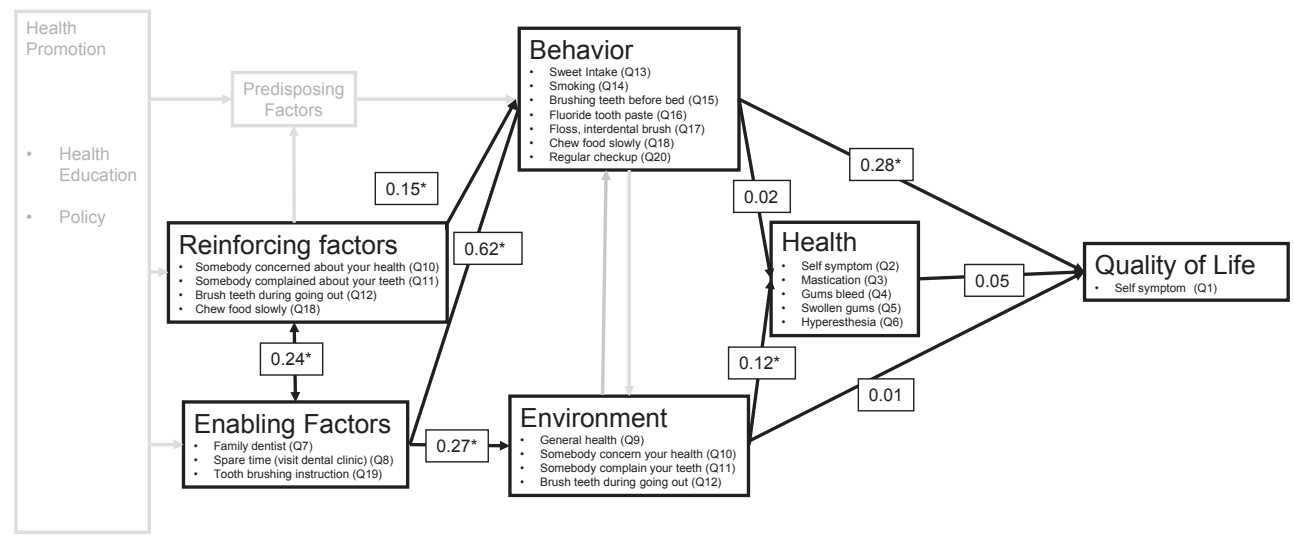

Fig. 2 Mapping out the items of the questionnaire in the framework of the PRECEDE-PROCEED model. Numbers show the coefficients. Path analysis was carried out by the first main principal component scores of each constituent of PRECEDE-PROCEED model. Fitness Indexes: RMSEA, 0.606; TLI, -1.581; CFI, 0.262; AIC, 730.873. * Indicate statistically significant $(P<0.05)$.

Table 2 Salivary levels of hemoglobin and lactate dehydrogenase against the response for PRECEDE-PROCEED model based questionnaire

\begin{tabular}{|c|c|c|c|c|c|c|c|c|c|c|c|c|c|}
\hline & & \multicolumn{6}{|c|}{$\mathrm{Hb}(\mu \mathrm{g} / \mathrm{mL})$} & \multicolumn{6}{|c|}{ LD (IU/L) } \\
\hline & & Mean & $\mathrm{SD}$ & Median & $\begin{array}{c}25 \\
\text { percentile }\end{array}$ & $\begin{array}{c}75 \\
\text { percentile }\end{array}$ & $P$ value & Mean & SD & Median & $\begin{array}{c}25 \\
\text { percentile }\end{array}$ & $\begin{array}{c}75 \\
\text { percentile }\end{array}$ & $P$ value \\
\hline \multirow[t]{2}{*}{ Self-symptom(Q1) } & - & 3.4 & 6.3 & 0.7 & 0.3 & 1.7 & \multirow{2}{*}{0.648} & 356.2 & 216.5 & 300 & 204 & 461 & \multirow{2}{*}{0.694} \\
\hline & + & 3.8 & 6.7 & 0.6 & 0.3 & 1.9 & & 365.7 & 224.4 & 293 & 202 & 470 & \\
\hline \multirow[t]{2}{*}{ Self-symptom (Q2) } & - & 3.5 & 6.4 & 0.6 & 0.3 & 1.7 & \multirow{2}{*}{0.052} & 356.4 & 215.5 & 295 & 204 & 459 & \multirow{2}{*}{0.176} \\
\hline & + & 5.2 & 8.1 & 0.9 & 0.4 & 5.9 & & 420.2 & 269.7 & 333 & 201 & 590 & \\
\hline \multirow[t]{2}{*}{ Mastication (Q3) } & - & 3.3 & 6.1 & 0.6 & 0.3 & 1.7 & \multirow[b]{2}{*}{0.207} & 355.2 & 216.0 & 293 & 204 & 438 & \multirow[b]{2}{*}{0.326} \\
\hline & + & 5.4 & 8.1 & 0.8 & 0.3 & 8.5 & & 393.0 & 242.7 & 329 & 196 & 543 & \\
\hline \multirow[t]{2}{*}{ Gums bleed (Q4) } & - & 2.3 & 4.9 & 0.6 & 0.3 & 1.3 & \multirow{2}{*}{$<0.001$} & 334.3 & 202.7 & 286 & 189 & 406 & \multirow{2}{*}{0.034} \\
\hline & + & 5.4 & 8.0 & 1.0 & 0.3 & 8.4 & & 398.0 & 238.8 & 326 & 210 & 529 & \\
\hline \multirow[t]{2}{*}{ Swollen gums (Q5) } & - & 3.4 & 6.2 & 0.6 & 0.3 & 1.7 & \multirow{2}{*}{0.239} & 346.4 & 209.6 & 293 & 199 & 425 & \multirow{2}{*}{0.056} \\
\hline & + & 4.7 & 7.8 & 0.8 & 0.3 & 3.2 & & 425.2 & 254.7 & 339 & 237 & 603 & \\
\hline \multirow[t]{2}{*}{ Hyperesthesia (Q6) } & - & 3.4 & 6.3 & 0.6 & 0.3 & 1.9 & \multirow{2}{*}{0.363} & 359.7 & 222.7 & 300 & 198 & 457 & \multirow{2}{*}{0.538} \\
\hline & + & 4.0 & 6.9 & 0.6 & 0.3 & 2.1 & & 364.7 & 219.6 & 304 & 207 & 475 & \\
\hline \multirow[t]{2}{*}{ Family dentist (Q7) } & - & 3.0 & 6.0 & 0.5 & 0.3 & 1.5 & & 350.4 & 214.9 & 293 & 201 & 436 & \\
\hline & + & 5.2 & 7.6 & 1.2 & 0.3 & 8.2 & 0.003 & 391.9 & 234.7 & 329 & 207 & 510 & 0.130 \\
\hline Spare time (visit dental clinic) (Q8) & - & 3.2 & 6.2 & 0.5 & 0.3 & 1.7 & & 340.1 & 207.9 & 278 & 191 & 455 & \\
\hline & + & 4.1 & 6.9 & 0.8 & 0.3 & 3.1 & 0.102 & 382.8 & 231.8 & 313 & 223 & 470 & 0.062 \\
\hline General health (Q9) & - & 3.5 & 6.4 & 0.6 & 0.3 & 1.7 & & 358.2 & 221.7 & 294 & 199 & 466 & \\
\hline & + & 5.4 & 8.2 & 1.1 & 0.4 & 7.0 & 0.183 & 413.6 & 208.8 & 372 & 254 & 473 & 0.104 \\
\hline Somebody concerned about your health (Q10) & - & 2.9 & 5.7 & 0.5 & 0.2 & 1.5 & & 341.7 & 199.6 & 292 & 204 & 427 & \\
\hline & + & 4.8 & 7.6 & 0.8 & 0.3 & 4.7 & 0.004 & 393.0 & 248.1 & 322 & 205 & 517 & 0.181 \\
\hline Somebody complained about your teeth (Q11) & - & 2.9 & 6.4 & 0.5 & 0.3 & 1.1 & & 318.8 & 216.3 & 268 & 172 & 416 & \\
\hline & + & 3.7 & 6.6 & 0.7 & 0.3 & 2.2 & 0.170 & 368.2 & 221.3 & 304 & 208 & 474 & 0.068 \\
\hline Brush teeth during going out (Q12) & - & 3.2 & 6.0 & 0.6 & 0.3 & 1.7 & & 345.5 & 190.3 & 316 & 202 & 467 & \\
\hline & + & 3.8 & 6.7 & 0.6 & 0.3 & 2.1 & 0.366 & 366.4 & 228.7 & 295 & 204 & 466 & 0.858 \\
\hline Sweet Intake (Q13) & - & 3.7 & 6.6 & 0.6 & 0.3 & 2.1 & & 341.4 & 192.4 & 320 & 179 & 461 & \\
\hline & + & 3.6 & 6.6 & 0.6 & 0.3 & 1.7 & 0.795 & 366.4 & 225.8 & 298 & 204 & 471 & 0.708 \\
\hline Smoking (Q14) & - & 3.4 & 6.3 & 0.6 & 0.3 & 1.8 & & 355.6 & 221.6 & 294 & 198 & 438 & \\
\hline & + & 4.7 & 7.5 & 0.8 & 0.4 & 2.3 & 0.050 & 385.6 & 218.6 & 313 & 220 & 510 & 0.151 \\
\hline Brushing teeth before bed (Q15) & - & 3.0 & 5.8 & 0.6 & 0.3 & 1.5 & & 340.8 & 192.9 & 293 & 206 & 406 & \\
\hline & + & 5.9 & 8.3 & 1.0 & 0.5 & 9.3 & 0.001 & 431.2 & 285.9 & 327 & 187 & 641 & 0.138 \\
\hline Fluoride tooth paste (Q16) & - & 3.0 & 5.9 & 0.6 & 0.2 & 1.6 & & 374.0 & 224.7 & 308 & 207 & 484 & \\
\hline & + & $\begin{array}{l}5.0 \\
4.2\end{array}$ & $\begin{array}{l}5.9 \\
7.1\end{array}$ & $\begin{array}{l}0.0 \\
0.7\end{array}$ & 0.3 & $\begin{array}{l}1.0 \\
2.6\end{array}$ & 0.039 & 351.2 & 217.7 & 286 & 200 & $\begin{array}{l}404 \\
447\end{array}$ & 0.434 \\
\hline Floss, interdental brush (Q17) & - & 2.7 & 6.0 & 0.4 & 0.2 & 1.1 & 0003 & 302.2 & 168.9 & 254 & 178 & 401 & \\
\hline & + & 3.9 & 6.7 & 0.7 & 0.3 & 2.3 & 0.003 & 378.4 & 230.9 & 313 & 218 & 479 & 0.014 \\
\hline Chew food slowly (Q18) & - & 5.1 & 7.8 & 0.8 & 0.2 & 7.2 & & 401.1 & 262.6 & 280 & 200 & 561 & \\
\hline & + & 3.3 & 6.2 & 0.6 & 0.3 & 1.6 & 0.837 & 351.5 & 207.9 & 302 & 206 & 424 & 0.536 \\
\hline Tooth brushing instruction (Q19) & - & 2.9 & 5.9 & 0.5 & 0.3 & 1.3 & & 344.1 & 205.8 & 293 & 201 & 422 & \\
\hline & + & 5.0 & 7.6 & 1.2 & 0.3 & 6.6 & 0.001 & 397.5 & 245.8 & 328 & 205 & 509 & 0.162 \\
\hline Regular checkup (Q20) & - & 3.2 & 6.3 & 0.5 & 0.3 & 1.4 & & 350.1 & 229.4 & 279 & 196 & 429 & \\
\hline & + & 4.0 & 6.8 & 0.8 & 0.3 & 2.8 & 0.067 & 371.3 & 214.3 & 320 & 210 & 478 & 0.171 \\
\hline
\end{tabular}

Results of saliva tests against the items of the standardized questionnaire. $P$ values were calculated by Mann-Whitney $U$ tests.

probability of a correct response to the item, whereas respondents with high ability have a high probability of a correct response. Items whose curves are relatively flat have low discrimination. Items with low discrimination are candidates to be dropped from the measurement instrument. The horizontal axis examines the subject's ability by test, and in this case, the axis shows unhealthy levels. Items related to general health were not suitable for evaluating oral health (Q2, Q9). Healthy subjects answered yes to Q11, and unhealthy subjects answered yes to Q3, Q5, Q14, and Q15.

The item information curve shows how precisely each item measures latent traits at various levels of the attribute. The greater the area under this curve, the more information the item possesses. Certain items may provide more information at low levels of the ability, whereas others may provide more information at higher levels of the ability. The highest item information was obtained by Q7, and the peak of the curve was shifted in the forward direction. Having or not having a family dentist was useful for distinguishing between unhealthy subjects and highly unhealthy subjects.

The items of the questionnaire used in this study corresponded to the components of PRECEDE-PROCEED model. Construct validity of the questionnaire was examined through path analysis. The structure of the questionnaire and results of the path analysis are shown in Fig. 2. Some of the paths could not be drawn due to the restrictions within the statistical model, and some of the paths were not statistically significant.

Statistically significant correlations were observed between the items of the questionnaire based on the PRECEDE-PROCEED model and salivary levels of biomarkers. Among the 20 items in questionnaire based on the PRECEDE-PROCEED model, 7 items were significantly associated with 


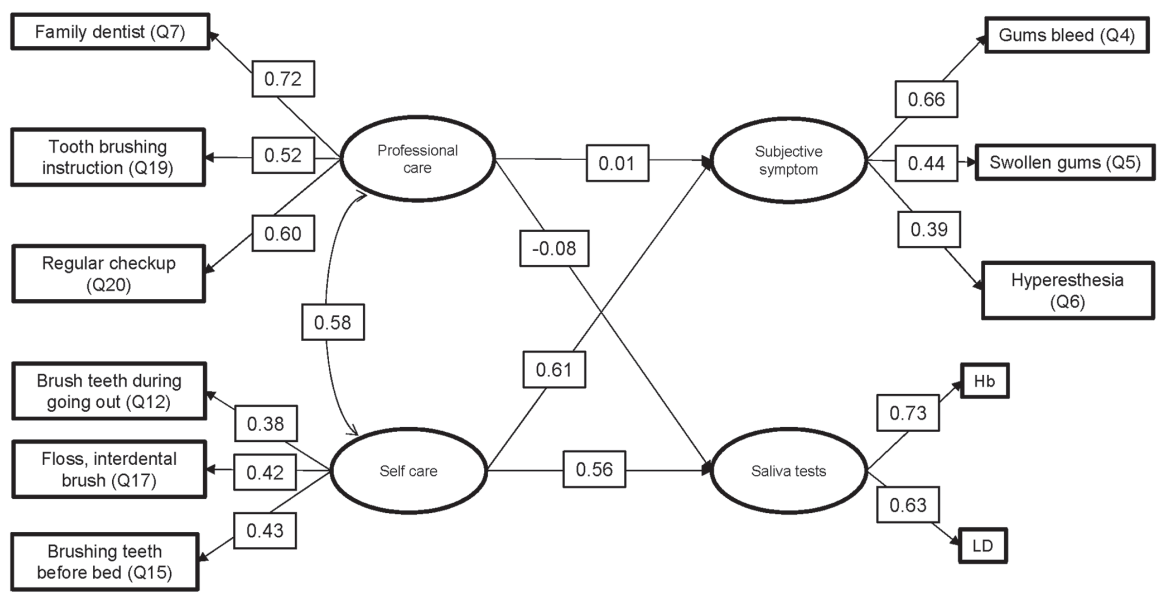

Fig. 3 Path diagram of professional care, self-care, subjective symptoms, and saliva tests. Ovals show the latent variables, and squares show the observed variables. Paths from professional care to subjective symptoms and to saliva tests were not statistically significant, and their coefficients were small. Fitness Indexes: RMSEA, 0.047; TLI, 0.87; CFI, 0.923; AIC, 141.883

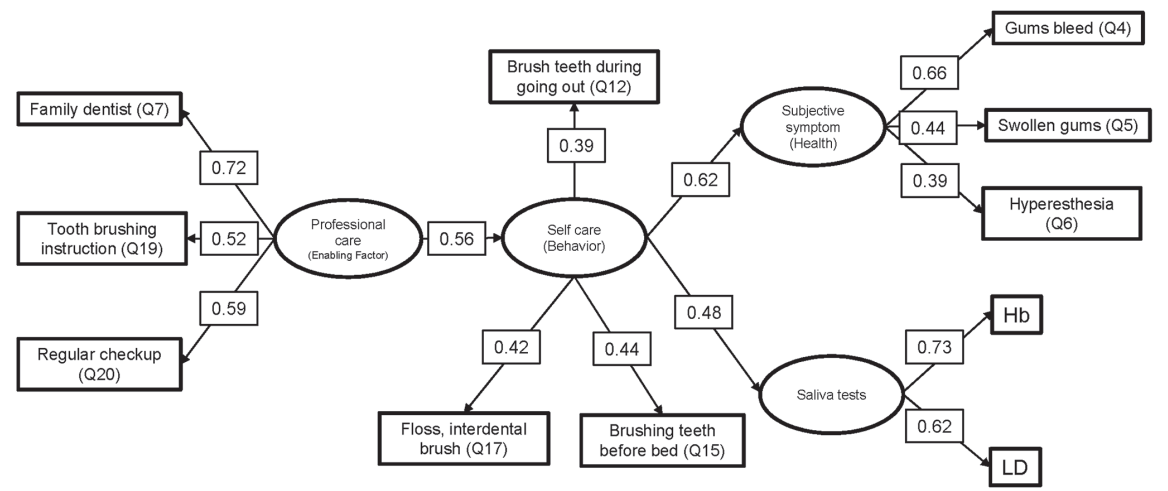

Fig. 4 Path diagram of professional care, self-care, subjective symptoms, and saliva tests. Ovals show the latent variables, and squares show the observed variables. This model suggested that professional care effects subjective symptoms and saliva tests through self-care instruction. Self-care instruction may be one of the important roles for the family dentist. Fitness Indexes: RMSEA, 0.045; TLI, 0.884; CFI, $0.928 ;$ AIC, 138.237

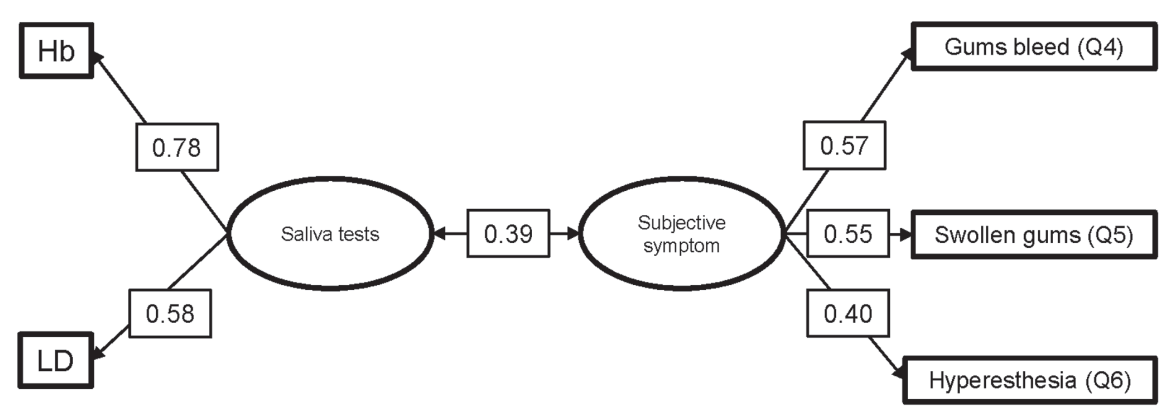

Fig. 5 The correlation between subjective symptoms and saliva tests. Ovals show the latent variables, and squares show the observed variables. The path between subjective symptoms and saliva tests was statistically significant. However, the coefficient was not large. Fitness Indexes: RMSEA, 0.070; TLI, 0.836; CFI, 0.956; AIC, 42.104

salivary $\mathrm{Hb}$, and 2 were significantly related to salivary LD. Bleeding during teeth brushing and daily use of dental floss or an interdental brush were significantly correlated with salivary $\mathrm{Hb}$ and LD (Table 2).

A conceptual scheme for oral disease screening using structural equation modeling based on the PRECEDE-PROCEED model and saliva test were constructed. First, the model shown in Fig. 3 was constructed. The paths from "professional care" to "subjective symptoms" and to "saliva tests" were not statistically significant ( $P=0.970$ and 0.585 , respectively). Thus, the model shown in Fig. 4 was constructed. All the paths were statistically significant including "professional care" to "subjective symptoms" and to "saliva tests." Statistically significant differences were observed between the path form "self-care" to "subjective symptom" and "saliva tests" $(P<$ $0.01)$. Additionally, the correlation between these two latent variables was assessed. The model illustrated in Fig. 5 had shown that the path between subjective symptoms and saliva tests was statistically significant; however, the value of the path coefficient was not large.

\section{Discussion}

Screening for individuals with, and at risk of oral disease, is a fundamental strategy for early detection, treatment, and prevention of onset and progression. While several methods of screening for oral diseases exist, for mass health checkups, it is important that the screening tools are cost effective. Despite having their limitations, some of the questionnaires have had their reliability and validity confirmed [3,10-12]. The questionnaire used in this study, based on PRECEDE-PROCEED model, was shown to be both reliable and valid.

Saliva tests for periodontal disease screening have been used in several studies $[4,8,9]$, and in these reports, the distributions of the salivary $\mathrm{Hb}$ were skewed; this is also consistent with previous report [13]. Saliva testing is a cost-effective method for oral disease screening, and the results of the tests are not subjective. In regard to the aforementioned screening methods, it is important to confirming the relationship between the two to establish a framework for incorporating them in oral health policy. 
Visiting a dentist for a routine dental checkup has been associated with improved oral health [14]. In Japan, the Ministry of Health and Labor recommend regularly attending checkups and cleanings at the family dentist. The Japanese government implemented a program for national health promotion in 2000 called Healthy Japan 21 and entered into phase two of the program in 2012. In the first phase, the government set the goal that more than $30 \%$ of people attend dental checkup at least once a year. In the second phase, the percentage was increased to $65 \%$ of people and lasts until 2022. According to this strategy, it is necessary to evaluate the role of family dentist.

The model in Fig. 2 indicates that visiting the dentist may not affect the subjective symptoms and result of the saliva tests directly. The subjective symptoms and result of the saliva tests may be affected mainly by the oral health self-care. The model in Fig. 4 more clearly suggests this. The path coefficient between "professional care" and "self-care" was significant; this model suggests that the self-care instruction may be one of the important roles for the family dentist.

Self-awareness of oral symptoms and results of saliva tests had significant but not strong correlations. The results of the saliva tests reflect only the inflammation of the gum. Subjective symptoms also include higher sensitivity of the teeth. The results of saliva test represent the presence of occult blood in saliva, and the presence of occult blood in saliva represents a sign of inflammation. This slight inflammation may not reflect one's self-awareness of oral symptoms [7]. This may be one of the reason strong correlation was not found between subjective symptoms and results of saliva tests. Thus, self-administrated questionnaires and saliva tests may not compete in the screening of oral diseases and should be used in conjunction with one another to provide more information during mass oral disease screening process.

\section{Acknowledgments}

This study was funded by a budget from the community health service of Kawasaki Dental Association.

\section{Conflict of interest}

The authors declared no potential conflict of interest with respect to the research, authorship, and/or publication of this article.

\section{References}

1. Boven GC, Raghoebar GM, Vissink A, Meijer HJ (2015) Improving masticatory performance, bite force, nutritional state and patient's satisfaction with implant overdentures: a systematic review of the literature. J Oral Rehabil 42, 220-233.

2. Lamster IB, Pagan M (2017) Periodontal disease and the metabolic syndrome. Int Dent J 67, 67-77.

3. Fukai K (2017) Oral health for achieving healthy longevity in an aging society - Evidence and Policy-. Int J Oral Health 13, 52-57.

4. Nomura Y, Tamaki Y, Tanaka T, Arakawa H, Tsurumoto A, Kirimura K et al. (2006) Screening of periodontitis with salivary enzyme tests. J Oral Sci 48, 177-183.

5. Kakuta E, Nomura Y, Naono Y, Koresawa K, Shimizu K, Hanada N (2013) Correlation between health-care costs and salivary tests. Int Dent J 63, 249-253.

6. Li Y, Cao J, Lin H, Li D, Wang Y, He J (2009) Community health needs assessment with precede-proceed model: a mixed methods study. BMC Health Serv Res, 9, 181.

7. Nomura Y, Shimada Y, Hanada N, Numabe Y, Kamoi K, Sato T et al. (2012) Salivary biomarkers for predicting the progression of chronic periodontitis. Arch Oral Biol 57, 413420.

8. Nomura Y, Okada A, Kakuta E, Gunji T, Kajiura S, Hanada N (2016) A new screening method for periodontitis: an alternative to the community periodontal index. BMC Oral Health 16, 64.

9. Okada A, Nomura Y, Sogabe K, Oku H, Sato Gillbreath A, Hino F et al. (2017) Comparison of salivary hemoglobin measurements for periodontitis screening. J Oral Sci 59, 63-69.

10. Ho AW, Grossi SG, Dunford RG, Genco RJ (1997) Reliability of a self-reported health questionnaire in a periodontal disease study. J Periodontal Res 32, 646-650.

11. Buhlin K, Gustafsson A, Andersson K, Håkansson J, Klinge B (2002) Validity and limitations of self-reported periodontal health. Community Dent Oral Epidemiol 30, 431-437.

12. Genco RJ, Falkner KL, Grossi S, Dunford R, Trevisan M (2007) Validity of self-reported measures for surveillance of periodontal disease in two western New York populationbased studies. J Periodontol 78, 1439-1454.

13. Yamada H, Okada A, MurataT, Uetani K, Kotoh M, Nishitsuji N et al. (2016) Prevalence of blood contamination in adult saliva and quantitative measurement of salivary hemoglobin level by using an anti-human hemoglobin monoclonal antibody. Asian Pac J Dent 16, 9-13.

14. Afonso-Souza G, Nadanovsky P, Chor D, Faerstein E, Werneck GL, Lopes CS (2007) Association between routine visits for dental checkup and self-perceived oral health in an adult population in Rio de Janeiro: the Pró-Saúde Study. Community Dent Oral Epidemiol $35,393-400$. 